\title{
Open loop doppler tracking in Chinese forthcoming Mars mission
}

\author{
Kun Shang ${ }^{1}$, Jinsong Ping ${ }^{1}$, Chunli Dai ${ }^{1}$, and Nianchuan Jian $^{1}$ \\ ${ }^{1}$ Shanghai Astronomical Observatory, \\ 80 Nandan Road, Shanghai 200030, China \\ email: shangkun@shao.ac.cn
}

\begin{abstract}
Using the radio telescopes in Chinese VLBI Network and the K5/VSSP32 VLBI system of NICT in Japan, we have developed algorithms that can extract open loop Doppler information from the Chang'E-1 and Mars Express radio tracking data. Our latest results indicate the Doppler accuracy of open loop three-way Doppler is about $1 \mathrm{~mm} / \mathrm{s}$ in 1 second integration time, relative to a $8.4 \mathrm{GHz}$ carrier. In the forthcoming joint Russian-Chinese Martian mission in 2009 , the current software algorithms and hardware performance will be improved, and we will attempt to use the high precision Doppler shift and phase information to test gravitation theories.
\end{abstract}

Keywords. celestial mechanics, space vehicles: instruments, techniques: radial velocities, planets and satellites: general

\section{Introduction}

Lunar and the planetary exploration is to recovery the origin of the Moon and the planets, to explore their evolution, and to look for the possible life in other planetary bodies. Since the beginning of the new century, Mars exploration has attracted the huge attention from space communities. As a beginner in this area, China has launched her 1st lunar orbiter Chang'E-1 (CE-1) successfully, and has got some new scientific results from this exploration. Beyond this, a joint Russian-Chinese Martian mission, YingHuo1 sub-satellite (YH-1) and Phobos-Grunt Spacecraft (FGSC), has been developed and promoted solidly. The two probes will be launched together in October 2009. After a successful launch, the joint spacecraft YH-1 and FGSC will be sent to a transfer orbit flying to Mars. After 10 - 11 months, the joint craft will arrive in the Martian system, and will be ejected into an equatorial orbit of $800 \mathrm{~km}$ periapsis altitude and $80,000 \mathrm{~km}$ apoapsis altitude, with a period of about 72 hours, inclination of $1^{\circ}-5^{\circ}$. The joint craft will fly in this orbit for about 3 circles, then they will be separated. FGSC will change its orbit in order to land on Phobos, and then take some soil $(0.1 \mathrm{~kg}-0.2 \mathrm{~kg})$ back to the Earth. YH-1 will free-fly in this large elliptical orbit for 1 year.

YH-1 is a small sub-satellite focused on investigating the Martian space environment and the solar wind-Mars interaction. It will be combined with FGSC to form a two-point measurement configuration in the Martian space environment. The two spacecrafts will also carry out satellite-to-satellite radio link, so as to study the Martian ionosphere by using radio occultation links at UHF.

\section{Open loop experiments and results}

Considering that the Chinese deep space tracking system is still under construction, there will not be any uplink system in China which can meet the power requirement of 
uplink communication for a distance of AU. To simplify and minimize the design, an $\mathrm{X}$-band receiver and X-band transmitter system have been adopted for onboard communication. There is not a common PLL transponder used for tracking. To solve the tracking and orbit determination problem, an USO-based one-way open loop concept will be used in YH-1, and the ground astronomical VLBI system (Chinese VLBI Network) will be used to receive the radio signal, so as to retrieve the Doppler information and then the differential Doppler information. The open loop Doppler observable will be applied in the positioning and orbit determination of $\mathrm{YH}-1$.

Chinese VLBI Network (CVN), composed of four radio telescopes, acts as the deep space tracking system in China. Four radio telescopes located in four long-distance stations with different sizes, including: Shanghai station with a $25 \mathrm{~m}$ telescope built in 1987 , Urumqi station with a $25 \mathrm{~m}$ telescope built in 1993 , Beijing station with a $50 \mathrm{~m}$ telescope built in 2006, and Kunming station with a 40m telescope built also in 2006. Recording devices have already been installed in two CVN stations - Shanghai and Urumqi. We use K5/VSSP32 sampling board as recording device, with a local H-maser atomic clock. K5/VSSP32 is a high-performance sampler dedicated to a geodetic VLBI system developed by the Kashima VLBI group of National Institute of Information and Communications Technology (NICT) in Japan.

During the nominal mission period of Chinese lunar orbiter CE-1, we used the threeway method to test the open loop tracking ability as a preview of future one-way method in YH-1 mission. Three-way method means that a tracking station sends uplink S-Band signal generated by $\mathrm{Rb}$ atomic clock to $\mathrm{CE}-1$, and then the transponder in the orbiter locks the uplink carrier wave and sends it back to the Earth. The reason why a one-way Doppler experiment was not carried in CE-1 is that there is no stable oscillator. Similar experiments have been done together with ESOC deep space tracking stations for tracking the Mars Express. The radio telescopes used as the receiver station in three-way doppler experiment are located in Shanghai and Urumqi.

A software receiver using the post-processing method is developed to retrieve the Doppler frequency from the data recorded in K5/VSSP32 sampler. The kernel of this algorithm is phase counting (Ping et al. 2001), which can extract the precise main frequency from the raw data. Comparing the calculated received main frequency with the original transmitted frequency, we obtain the frequency shift in a certain integral time. The Doppler Effect presents the relationship between the frequency shift and the radial velocities.

$$
f_{R} \approx\left(1-\frac{v_{1}}{c}\right)\left(1-\frac{v_{2}}{c}\right) M \cdot f_{T} \approx\left(1-\frac{v_{1}+v_{2}}{c}\right) M \cdot f_{T}
$$

Where, $f_{R}$ represents the calculated received frequency, $f_{T}$ represents the original transmitted frequency, $v_{1}$ represents the range rate of the transmitting station and spacecraft, $v_{2}$ represents the range rate of spacecraft and receiving station, and $M$ represents the transmit ratio. Define the average of $v_{1}$ and $v_{2}$ as the three-way velocity, which means $v_{3 w}=\left(v_{1}+v_{2}\right) / 2$. Figure 1 and Figure 2 show the open loop three-way Doppler results of CE-1 mission and Mars Express mission, including the three-way velocity and the residuals after orbit determination.

\section{Prospects}

At present, the open loop Doppler accuracy of the Chinese tracking system is about $3 \mathrm{~mm} / \mathrm{s}$ in S-band and $1 \mathrm{~mm} / \mathrm{s}$ in X-band, both in 1 second integration time. Compared with the result of DSN in JPL (Thornton 2002), the current accuracy of China is 

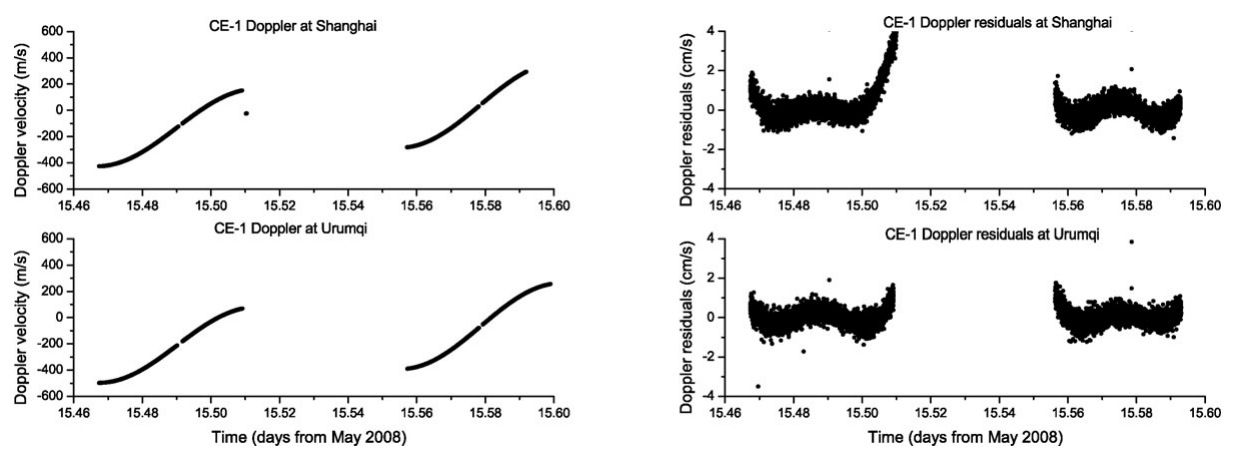

Figure 1. Open loop three-way Doppler result of CE-1 mission on 15 May 2008. The left panel shows the three-way Doppler velocity of Shanghai and Urumqi station. The right panel shows the Orbit Determination Doppler residual. The r.m.s of 1 second integration time is about $3 \mathrm{~mm} / \mathrm{s}$, relative to a $2.3 \mathrm{GHz}$ carrier.
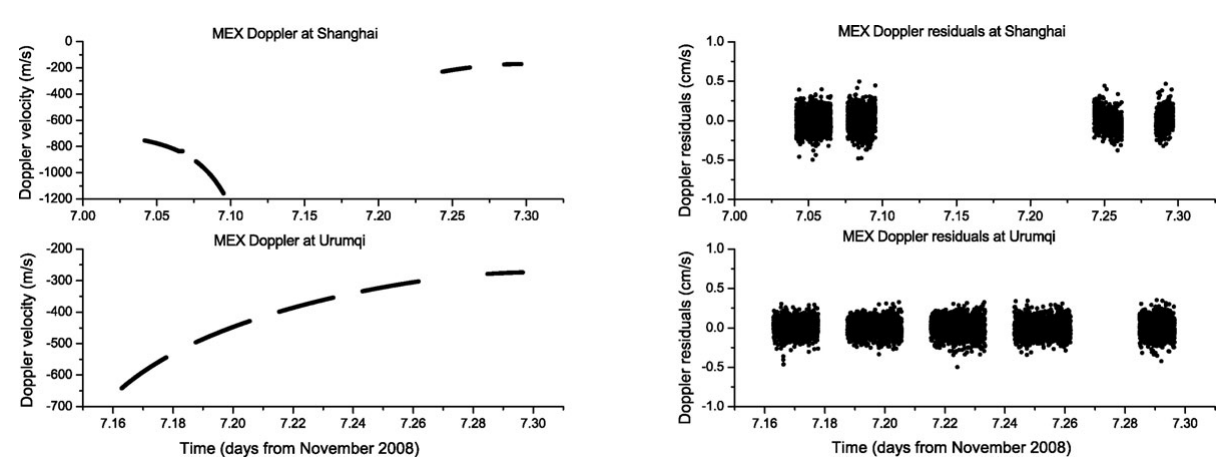

Figure 2. Open loop three-way Doppler result of MEX mission on 7 November 2008. The left panel shows the three-way Doppler velocity of Shanghai and Urumqi station. The right panel shows the Orbit Determination Doppler residual. The r.m.s of 1 second integration time is about $1 \mathrm{~mm} / \mathrm{s}$, relative to an $8.4 \mathrm{GHz}$ carrier.

better than that of JPL in 1980s but worst than that of JPL in 1990s. The limit of accuracy is restricted by the size of the radio telescopes in CVN. In 2014, a $65 \mathrm{~m}$ radio telescope will be built by Shanghai Astronomical Observatory, which will improve the sensitivity and accuracy in the Chinese deep space missions, especially the open loop Doppler tracking accuracy. High accuracy Doppler tracking can be used for the test of gravitational theories, such as testing the PPN parameter $\gamma$ (Will 1993). Considering the large elliptical orbit of the YH-1 satellite, other relativistic Doppler shift such as second order Doppler shift and gravitational shift can also be tested.

\section{Acknowledgements}

This work was supported by the VLBI system of Chinese Academic of Science and GEODYN software provided by NASA GFSC.

\section{References}

Ping, J. S., Frank, W., \& Yusuke, K. 2001, Journal of Planetary Geodesy, 36, 15-22

Thornton, C. L. \& Border, J. S. 2002, Radiometric Tracking Techniques for Deep-Space Navigation (Pasadena, California: JPL Publication), p. 9-46

Will, C. W. 1993, Theory and Experiment in Gravitational Physics (Cambridge: Cambridge University Press) 